\section{Microbial co-culture: harnessing intermicrobial signaling for the production of novel antimicrobials}

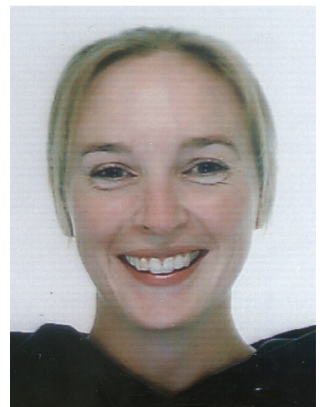

Suzy C Moody*

\author{
"In this editorial, we approach the world of \\ microbial signaling from a fresh angle and discuss \\ how intermicrobial communication might be used \\ to our advantage in the pharmaceutical field.”
}

Bacteria and fungi are masters of communication. They engage in constant information transfer with their neighbors, competitors and hosts. This elaborate 'social networking' is mediated by small bioactive molecules that are released from and received by cells, and that govern the cellular responses essential for survival in heterogeneous environments. As interest in microbial communication increases, scientists are rapidly appreciating how great a role this intermicrobial signaling plays in the microbial world, and how the elucidation of some of the pathways and molecules involved could impact on advances in medical microbiology. The global health emergency associated with the antibiotic resistance and the urgent need to produce new pharmaceutical therapeutics is well acknowledged [1]. Research in this area has revealed how responsive pathogens are in their ability to alter cellular biochemistry and the production profile of specific metabolites to enhance virulence, evade detection and prevent eradication $[2,3]$. In this editorial, we approach the world of microbial signaling from a fresh angle and discuss how intermicrobial communication might be used to our advantage in the pharmaceutical field. The fundamentally heterogeneous nature of many microbial niches suggests that mining communities of microbes for novel antimicrobials may be an important avenue to explore.

Many clinically important antibiotics, and numerous other pharmaceutically useful compounds, are produced by Actinomycetes, mainly from the genus Streptomyces. For example, the antituberculosis antibiotic streptomycin is produced by Streptomyces griseus [4] and tacrolimus, produced by $S$. tsukubaensis [5], is an important immune suppressant used for reducing transplant rejection. Antibiotics and other biomedically useful natural products are widely thought of as secondary or specialized metabolites, as they often appear to play no part in the primary metabolism of the producing organism but instead confer a survival advantage under certain environmental conditions [6]. It is currently believed that antibiotic compounds rarely, if ever, accumulate to killing concentrations in the natural environment and that these small bioactive molecules may instead act as signaling molecules themselves [7]. Indeed, several studies have shown how antibiotics at sub-lethal concentration can
*Department of Biosciences, College of Science, Swansea University, Singleton Park, Swansea, SA2 8PP, UK; s.c.moody@swansea.ac.uk

\section{KEYWORDS}

- antibiotic discovery $\bullet$ co-culture - fungal ecology • secondary metabolites $\bullet$ signaling

- Streptomyces 


\section{"Changing our view of microbes and their extensive intercellular signaling potential, and creating the right conditions for cells to communicate with their neighbors, may lead to a new generation of bioactive molecules being revealed.”}

have wide-ranging impacts on bacterial gene expression [8] and multiple gene promoters in clinically relevant species such as Staphylococcus aureus [9]. Additionally, the direct application of certain antibiotics (e.g., hormaomycin) has also been reported to activate biosynthetic pathways in treated streptomycete cells [10]. The application of this antibiotic-stimulated production of novel antimicrobials is limited, however, partly by the requirement for purified forms of antibiotic and partly by a lack of knowledge regarding which antibiotics will elicit production of the most pharmaceutically important metabolites.

The ever expanding volume of genomic sequencing data continues to facilitate identification of potential new antibiotic biosynthetic pathways using bioinformatic tools. Key biosynthetic protein families such as non-ribosomal peptide synthases and cytochrome P450 enzymes have sufficient amino acid identity that they are easily identified by sequence analysis, and this has led to the discovery of many new biosynthetic pathways that might be harnessed for the production of new drugs $[11,12]$. Significant challenges remain, however, in the identification of cryptic metabolic pathways that may lack conserved motifs and genetic identifiers, and in discovering precisely how to activate production of these specialized metabolites in vitro. It is in this context that a clearer understanding of microbial ecology, concomitant with a systems biology approach (integrating transcriptomic, proteomic and metabolomics data) could prove invaluable for new antimicrobial discovery.

Co-culture methodology provides one strategy of promise. It follows that by growing potential antibiotic-producing organisms in mixed culture, we might be able to encourage expression of cryptic pathways - and production of bioactive molecules - as the cells respond to signaling molecules in their environment. One advantage of this methodology is that prior knowledge of the signaling mechanism is not required, although better understanding of microbial community interactions, particularly the regulatory processes involved, will undoubtedly aid future experimental design.

Evidence for the co-culture-based approach is steadily growing. A recent paper described the changes to the secreted metabolome of Streptomyces coelicolor when paired with five other actinomycete soil inhabitants in turn. By combining the power of combining two techniques - nanospray desorption electrospray ionization (Nano-DESI) and matrix-assisted laser desorption ionization-time of flight (MaldiTOF) imaging mass spectrometry (IMS) - the co-culture approach revealed many novel compounds being produced by $S$. coelicolor. These included 12 desferrioxamines (iron-chelating siderophores) with acyl side chains of different lengths, the presence of which suggests the activity of intricate tailoring mechanisms during biosynthesis. Furthermore, each co-culture experiment resulted in a unique secondary metabolite 'fingerprint' with the combination of molecules being produced specific to particular microbe pairings [13]. Co-culture approaches for enhancing secondary metabolite production have been successful with other microbes. For example, the fungal endophyte Paraconiothyrium inhabits yew trees and is responsible for production of the potent anticancer drug paclitaxel. The production of paclitaxel was initially believed to be stimulated by metabolites produced by the plant. Recent research has challenged this, however, demonstrating instead that the stimulants are derived from other microbial members of the same ecological niche. Co-culturing Paraconiothyrium with the bark fungus Alternaria led to a small increase in paclitaxel production. Addition of a further fungal community member, Phomopsis, to the culture led to an eightfold increase in production of paclitaxel [14]. This research underscores why we should not overlook the natural habitat and ecology of microbes and how understanding microbial processes outside the laboratory could greatly enhance the production of pharmaceuticals.

The well-characterized fungal pathogen, Candida albicans, has recently been found to produce a number of known and novel volatile compounds (e.g., dihydrofarnesol and farnesol) when grown in vitro with other common skin commensal fungi such as Trichophytum rubrum. While farnesol is well known as a metabolite produced by $C$. albicans, dihydrofarnesol production only appears to be triggered by the presence of $T$. rubrum. As a novel volatile compound, dihydrofarnesol was subsequently assessed for antifungal activity and found to be more potent than fluconazole (a frontline clinical antifungal agent), showing higher antimicrobial activity against $T$. rubrum [15]. This is encouraging, not least because of the emergence of multiple azole resistance in clinical mycoses and the paucity of new antifungal therapeutics on the horizon [16]. 
While some interactions appear to depend on diffusible signals [15], in some circumstances coculture appears to trigger production of novel antibiotic compounds without evidence of diffusible signaling molecule involvement. For example, co-culture of mycolic acid coryneform bacteria such as Tsukumurella, Rhodococcus and Corynebacteria with a multitude of Streptomyces species is reported to induce biosynthetic pathways with the concomitant production of novel antimicrobials. Co-culture with Tsukumurella pulmonis or Corynebacteria glutamicum was found to activate a novel pathway in a species identified as Streptomyces endus S-522, giving rise to a new heterocyclic chromophore-containing antibiotic named alchivemycin A. Monoculture of $S$. endus did not yield the same compound, nor did exposure to filter-sterilized media from mycolic acid bacterial culture. The production of alchivemycin A therefore appears to require cell:cell contact between $S$. endus and the coryneform bacteria. It is possible that mycolic acid, bound to the outermost cell wall layer of the coryneform bacteria, may act as a signal to streptomycete cells triggering an intracellular signaling cascade that leads to activation of previously cryptic pathways. It is noteworthy that co-culture of T. pulmonis with over 100 different streptomycete species revealed increased production of known secondary metabolites in $54 \%$ of streptomycetes, while new metabolites were produced by $36 \%$ [17]. This is an exciting result that promises to provide new insights for the discovery and production of novel antimicrobials.

Several wood decay basidiomycetes are known to produce a variety of enzymes and secondary metabolites in monoculture. Yet wood decay fungi grow in complex mixed communities in the environment. Mimicking those interactions through co-culture of species such as Trametes versicolor, Bjerkandera adusta and Hypholoma fasciculare has shown marked changes in production of such specialized metabolites. For example, the production of enzymes such as laccase (phenoloxidase), which is used as an industrial catalyst and for bioremediation, is greatly increased [18]. This enhanced production is reported to be limited to the interaction zone between $T$. versicolor and its fungal competitor, suggesting again that close contact between mycelia plays an important role in altering the expression patterns of biosynthetic pathways. T. versicolor is also known to produce a variety of pharmaceutically useful compounds such as PSK (a polysaccharide with potent anti-tumor and anti-HIV properties) and PSP (an immune modulator) [19]. The biosynthetic pathways for compounds such as these may be activated in the presence of other fungi from the same ecological niche, and genes related to signaling are often also switched on in the same time frame [20]. The exact mechanisms through which signals are recognized and interpreted, and how T. versicolor effects responses to specific stimuli remains to be elucidated.

Changing our view of microbes and their extensive intercellular signaling potential, and creating the right conditions for cells to communicate with their neighbors, may lead to a new generation of bioactive molecules being revealed. With the prevalence of the 'ESKAPE' pathogens showing no sign of decline, new antimicrobials are desperately needed to reduce morbidity and mortality associated with these infective agents [1]. Co-culture of organisms we already work with is providing a rapid approach to switching on novel metabolite pathways. In furthering our understanding of the complexity of microbial ecology and appreciating the potentially ubiquitous nature of intermicrobial social networking, we may be able to harness their interactions in co-culture for enhancing our own pharmacopeia.

\section{Acknowledgements}

The author thanks CM Hull for her advice on the manuscript.

\section{Financial \& competing interests disclosure}

SC Moody is funded by the Natural Environment Research Council. SC Moody has no other relevant affiliations or financial involvement with any organization or entity with a financial interest in or financial conflict with the subject matter or materials discussed in the manuscript apart from those disclosed.

No writing assistance was utilized in the production of this manuscript.

\section{References}

1 Bogan C, Marchaim D. The role of antimicrobial stewardship in curbing carbapenem resistance. Future Microbiol. 8(8), 979-991 (2013).

2

Cook LC, LaSarre B, Federle MJ.

Interspecies communication among commensal and pathogenic streptococci. MBio. 4(4), pii: e00382-13 (2013).
3 Vega M, Allison KR, Samuels AN, Klempner MS, Collins JJ. Salmonella typhimurium intercepts Escherichia coli signaling to enhance antibiotic tolerance. Proc. Natl Acad. Sci. USA 110(35), 14420-14425 (2013). 


\section{EDITORIAL Moody}

4 Ohnishi Y, Ishikawa J, Hara $\mathrm{H}$ et al. Genome sequence of the streptomycin-producing microorganism Streptomyces griseus IFO 13350. J. Bacteriol. 190(11), 4050-4060 (2008).

5 Barreiro C, Prieto C, Sola-Landa A et al. Draft genome of Streptomyces tsukubaensis NRRL 18488 , the producer of the clinically important immunosuppressant tacrolimus (FK506). J. Bacteriol. 194(14), 3756-3757 (2012).

6 Challis GL, Hopwood DA. Synergy and contingency as driving forces for the evolution of multiple secondary metabolite production by Streptomyces species. Proc. Natl Acad. Sci. USA 25(100 Suppl. 2), 14555-14561 (2003).

7 Davies J. Are antibiotics naturally antibiotics? J. Ind. Microbiol. Biotechnol. 33(7), 496-499 (2006).

8 Goh EB, Yim G, Tsui W, McClure J, Surette MG, Davies J. Transcriptional modulation of bacterial gene expression by subinhibitory concentrations of antibiotics. Proc. Natl Acad. Sci. USA 99(26), 17025-17030 (2002).

9 Mesak LR, Miao V, Davies J. Effects of subinhibitory concentrations of antibiotics on SOS and DNA repair gene expression in Staphylococcus aureus. Antimicrob Agents Chemother. 52(9), 3394-3397 (2008).
10 Andres N, Wolf H, Zahner H. Hormaomycin, a new peptide lactone antibiotic effective in inducing cytodifferentiation and antibiotic biosynthesis in some Streptomyces species. Z. Naturforsch. 45c, 851-855 (1990).

11 Moody SC, Zhao B, Lei L et al. Investigating conservation of the albaflavenone biosynthetic pathway and CYP170 bifunctionality in streptomycetes. FEBS J. 279(9), 1640-1649 (2012).

12 Claesen J, Bibb MJ. Biosynthesis and regulation of grisemycin, a new member of the linaridin family of ribosomally synthesized peptides produced by Streptomyces griseus IFO 13350. J. Bacteriol. 193(10), 2510-2516 (2011).

13 Traxler MF, Watrous JD, Alexandrov T, Dorrestein PC, Kolter R. Interspecies interactions stimulate diversification of the Streptomyces coelicolor secreted metabolome. MBio. 4(4), pii: e00459-e00413 (2013).

14 Soliman SS, Raizada MN. Interactions between co-habitating fungi elicit synthesis of Taxol from an endophytic fungus in host Taxus plants. Front. Microbiol. 4, 1-14 (2013).

15 Brasch J, Horter F, Fritsch D, BeckJendroschek V, Tröger A, Francke W. Acyclic sesquiterpenes released by Candida albicans inhibit growth of dermatophytes. Med. Mycol. doi:10.3109/13693786.814174 (2013) (Epub ahead of print).

16 Hull CM, Purdy NJ, Moody SC. Mitigation of human-pathogenic fungi that exhibit resistance to medical agents: Can clinical antifungal stewardship help? Future Microbiol. (2014) (In Press).

17 Onaka H, Mori Y, Igarashi Y, Furumai T. Mycolic acid-containing bacteria induce natural-product biosynthesis in Streptomyces species. Appl. Environ. Microbiol. 77(2), 400-406 (2011).

18 Hiscox J, Baldrian P, Rogers HJ, Boddy L. Changes in oxidative enzyme activity during interspecific mycelial interactions involving the white-rot fungus Trametes versicolor. Fungal Genet. Biol. 47(6), 562-571 (2010).

19 Zjawiony JK. Biologically active compounds from Aphyllophorales (polypore) fungi. J. Nat. Prod. 67(2), 300-310 (2004).

20 Eyre C, Muftah W, Hiscox J et al. Microarray analysis of differential gene expression elicited in Trametes versicolor during interspecific mycelial interactions. Fungal Biol. 114(8), 646-660 (2010). 